\title{
Propuesta metodológica para el análisis de blogs periodísticos
}

\author{
Juliana Colussi Ribeiro*
}

\section{Resumen}

Este trabajo presenta una propuesta metodológica para el análisis de blogs periodísticos (j-blogs) integrados en la Web de medios de Comunicación. La propuesta se origina de la observación y del análisis comparativo de diez blogs periodísticos, labor desarrollada a lo largo del periodo de investigación doctoral. Se ha combinado una serie de técnicas de investigación, tales como el análisis de contenido Web, la observación sistemática abierta, la investigación de campo y la entrevista en profundidad. Gracias a su capacidad de amplitud y profundidad, el análisis de contenido Web se revela como una técnica investigativa que permite verificar los elementos propios del blog así como los aspectos periodísticos del contenido publicado. Las demás técnicas tienen una función complementaria. A través de la aplicación de la propuesta metodológica detallada en este artículo, se ha podido verificar diferentes aspectos relativos al formato, al contenido y a los elementos periodísticos de los $j$-blogs.

Palabras clave: Blog periodístico. Ciberperiodismo. Metodología. Análisis.

\section{Methodological proposal for the analysis of journalistic blogs Abstract}

This paper presents a methodology proposal for the analysis of journalistic blogs ( $\mathrm{j}$-blog) hosted on media's Web. The proposal stems from the observation and the comparative analysis of ten journalistic blogs, during the period of doctoral research. Refers to the combined use of investigative techniques, such as the Web content analysis, open systematic observation, field word and in-depth interview. Thanks to its capacity of breadth and depth, the Web content analysis reveals itself as a research technique that allows the researcher to verify the proper elements of the blog as the journalistic aspects of the content published. The other techniques have a complementary function. Through the application of

* Profesora doctora investigadora de Postdoctorado del Laboratório de Comunicação Online (LabCom), do Instituto Coordenador da Investigação (ICI), da Universidade da Beira Interior, Covilhã - Beira Interior, Portugal. E-mail: julianacolussi@hotmail.com 
the methodology detailed in this article, has been able to verify different aspects of the format, content and journalistic elements of the $\mathrm{j}$-blogs.

Keywords: Journalistic blog. Online journalism. Methodology. Analysis.

\section{Proposta metodológica para a análise de blogs jornalísticos Resumo}

Este artigo apresenta uma proposta metodológica para a análise de blogs jornalísticos (j-blogs) hospedados em veículos de Comunicação na Web. Tal proposta origina-se a partir da observação e análise comparativa de dez blogs jornalísticos, realizadas durante o período de pesquisa doutoral. Refere-se ao uso combinado de uma série de técnicas de investigação, tais como a análise de conteúdo Web, a observação sistemática aberta, a pesquisa de campo e a entrevista em profundidade. Graças a sua capacidade de amplitude e profundidade, a análise de conteúdo Web revela-se como uma técnica de pesquisa que permite ao pesquisador verificar tanto os elementos próprios do blog como dos aspectos jornalísticos do conteúdo publicado. As demais técnicas têm uma função complementar. Ao aplicar a proposta metodológica descrita neste artigo, pudemos verificar diferentes aspectos relativos ao formato, conteúdo e elementos jornalísticos dos j-blogs.

Palavras chave: Blog jornalístico. Jornalismo digital. Metodologia. Análise.

\section{Introducción}

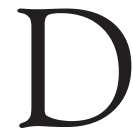

esde que los blogs ganaron protagonismo en internet, sobretodo a partir de 2003, cuando los corresponsales de diferentes medios de comunicación utilizan esta herramienta para publicar actualidades sobre la Guerra de Iraq (RECUERO, 2003), los académicos se han dedicado a investigar la relación entre blogs y periodismo (SINGER, 2005; ROBINSON, 2006; ARAÚJO, 2006; NOGUERA VIVO, 2006; DOMINGO; HEINONEN, 2008; FOLETTO, 2009) mediante el uso de técnicas de investigación, que no necesariamente responden de forma satisfactoria las preguntas relativas al análisis de los elementos periodísticos de estos blogs. Pensando en este problema de investigación, este trabajo presenta una propuesta metodológica para el análisis de blogs periodísticos (j-blog) que están integrados en la Web de medios de comunicación.

Conviene subrayar que este artículo resulta de una investigación doctoral, en la que se desarrolló esta metodología para el 
análisis comparativo de diez j-blogs sobre política integrados en la Web de periódicos de referencia de Brasil y España (COLUSSI RIBEIRO, 2013). Se trata de un estudio empírico en el cual se ha utilizado la observación sistemática de los blogs periodísticos para la construcción de las categorías de análisis - descritas adelante. Se ha utilizado la presente metodología para analizar 977 posts publicados en el periodo de dos semanas en diez j-blogs.

A lo largo del desarrollo de la propuesta metodológica han surgido algunas preguntas de investigación: ¿Cómo delimitar las categorías de análisis considerando las características del blog como herramienta o medio sin olvidarse de los elementos periodísticos? ¿El análisis de contenido Web es suficiente para responder las preguntas de investigación? ¿Cuál debe ser el periodo de análisis? ¿Cuántos posts se analizará?

Con el objetivo de contribuir para la definición de categorías específicas para el análisis de blogs periodísticos, se ha diseñado una propuesta metodológica que combina diferentes técnicas de investigación, tales como la observación sistemática abierta, la investigación de campo, la entrevista en profundidad y el análisis de contenido Web (HERRING, 2010). Además de las técnicas de investigación propuestas, el presente estudio pretende ampliar el debate en torno a las subcategorías de análisis, que en este caso forman parte de los siguientes apartados del análisis de contenido Web: 1) Análisis de formato de los blogs, 2) Análisis de contenido y 3) Análisis periodístico.

La primera parte del análisis de contenido Web, que se denomina análisis de formato de los blogs, examina los elementos que están propiciando la evolución con respecto al diseño y a la estructura de los blogs periodísticos. Esta parte del análisis se convierte en un aspecto fundamental para comprender los elementos personalizados por los periodistas-blogueros. Por ello, se divide en cuatro grandes categorías: presentación, contenido, accesibilidad y elementos.

El segundo apartado, referente al análisis de contenido, corresponde a un análisis cuantitativo, en el cual se da a conocer algunos datos referentes a los blogs periodísticos, tales como el promedio de entradas por semana, el promedio de palabras por 
post, el promedio de palabras por título de cada entrada, el promedio de comentarios por entrada, de hiperenlaces por entrada. La interpretación de los datos del análisis de contenido Web sirve como complemento para analizar, sobre todo, el estilo de redacción y el nivel de interactividad de los periodistas-blogueros con los usuarios y entre los usuarios.

Por último se encuentra el análisis periodístico, que se refiere al análisis de los elementos propiamente periodísticos, con el objetivo de conocer el estilo de redacción, la narrativa y otros elementos del contenido de los j-blogs. Para ello, se indica el análisis de diez categorías: 1) Manual de estilo, 2) Origen del contenido, 3) Géneros de los posts, 4) Estructura del texto, 5) Narrativa de los posts, 6) Gramática y ortografía, 7) Número y tipos de fuentes informativas, 8) Número, tipo y destino de los enlaces hipertextuales, 9) Fomento de la participación y 10) Capacidad de producción.

\section{Técnicas de investigación}

De acuerdo con lo expuesto en el cuadro 1, se recomienda el uso de las siguientes técnicas de investigación para llevar a cabo la recogida de datos de los j-blogs elegidos como objetos de estudio: 1) revisión bibliográfica; 2) investigación de campo; 3) observación sistemática abierta; 4) entrevista en profundidad; y 5) análisis de contenido Web. A continuación, se detalla la aplicación de cada técnica de investigación.

Para diseñar la presente propuesta metodológica, cuatro referencias bibliográficas han servido como una especie de guía para la elección de las técnicas investigativas: Cibermedios: métodos de investigación, de Díaz y Palácios (2009); Web contentanalisys: expandingtheparadigm, de Herring (2010); Métodos de pesquisa para Internet, editado por Fragoso et al. (2011); y Ferramenta para Análise de Blogs em Cibermeios, de Meso et al. (2011). 
Cuadro 1Técnicas de investigación propuestas para el análisis de j-blogs

\section{Técnicas de investigación}

Documentación indirecta

Documentación directa

Observación directa intensiva

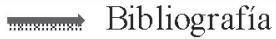

Investigación de campo

Observación sistemática abierta

Observación directa extensiva

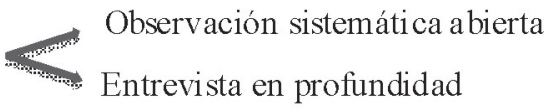

Análisis de contenido Web

Gracias a la capacidad de amplitud y profundidad del análisis de contenido Web aplicado a los blogs (HERRING, 2010), se ha optado por esta técnica de investigación que abarca diferentes tipos de categorías, como los temas de los posts, los comentarios, los enlaces, los recursos multimedia etc. Con el objetivo de complementar el análisis de contenido Web y, considerando que la investigación empírica en esta materia es aún "incipiente y las aproximaciones metodológicas son muy diversas" (DÍAZ NOCI; PALÁCIOS, 2009, p.85), se recomienda la utilización de otras dos técnicas de investigación: la observación sistemática y la entrevista. La observación sistemática contribuye a investigaciones empíricas sobre ciberperiodismo (DÍAZ NOCI; PALÁCIOS, 2009). A su vez, la entrevista funciona como una técnica de investigación complementaria, ya que puede añadir datos e informaciones de ámbito cualitativo al trabajo investigativo. En realidad, se trata de una combinación de una serie de técnicas investigativas, identificadas separadamente por Fragoso et al. (2011) en estudios anteriores sobre blogs.

La revisión bibliográfica, que estructura el marco teórico del trabajo de investigación, incluye libros y artículos académicos sobre ciberperiodismo y blogs. Este apartado ofrece una base teórica para el trabajo y se adapta tanto al objeto de estudio como a los objetivos e hipótesis de la investigación. 
En cuanto a la investigación de campo, se propone el uso de la del tipo exploratorio-descriptivo, ya que tiene por objetivo describir el proceso de generación de contenidos en los j-blogs, incluyendo la narrativa, la redacción, el rigor periodístico y la interacción entre profesionales y usuarios. Se trata de un tipo de investigación que permite la combinación de una variedad de procedimientos de recogida de datos. En este caso, se adoptan la entrevista en profundidad (DUARTE, 2005), la observación sistemática abierta (DÍAZ NOCI; PALÁCIOS, 2009) y el análisis de contenido Web (HERRING, 2010). Conviene recordar que Quandt (2008) también recomienda la combinación de esas técnicas en estudios comparativos.

Como bien señalan Marconi y Lakatos, este tipo de investigación empírica consiste en "formular cuestiones con triple finalidad: desarrollar hipótesis, aumentar la familiaridad del investigador con un ambiente, hecho o fenómeno, para la realización de un estudio futuro más preciso, o modificar o clarificar conceptos" (2006, p.190). De ese tipo de investigación empírica se suele obtener descripciones tanto cuantitativas como cualitativas del objeto de estudio. Además, el investigador debe conceptuar las relaciones entre las propiedades del fenómeno, hecho o ambiente observado.

\section{Observación de los j-blogs}

Antes del inicio de la observación sistemática del objeto de estudio, se sugiere seleccionar la muestra de los blogs que serán analizados. El uso de la observación sistemática abierta tiene como propósito contribuir para el proceso de delimitación de las categorías de análisis referente al formato, contenido y elementos periodísticos. Sin la etapa de la observación, las categorías podrían no reflejar todos los elementos que componen los blogs periodísticos que formarán parte del análisis.

En este caso, se trata de una observación directa intensiva, de acuerdo con la acepción de Marconi y Lakatos (2006). Para la realización de la observación, se consideró la tendencia de los estudios actuales de periodismo, en los cuales el término observación tiene 
un significado especial. Conforme señala Quandt $(2008)^{1}$, este término se refiere a un método científico que tiene implicaciones más allá de la recopilación de datos. Para cualquier aplicación del método, el investigador debe considerar tres características que diferencian el enfoque científico de la observación cotidiana. El proceso de la observación debe: 1) servir a un propósito de investigación formulado, 2) ser planificado de forma sistemática y 3) registrar los datos de manera sistemática.

Una de las principales contribuciones de Quandt corresponde a la descripción de los escenarios de la investigación periodística en que se aplica la observación. Entre los escenarios más comunes, están la observación en las redacciones de noticia, lo que denomina análisis de entrada-proceso-salida (la información bruta, el proceso de edición y la publicación del contenido) y los estudios de usabilidad (utilizados para realizar estudios de audiencia en medios de comunicación digital).

Para observar los j-blogs, se puede utilizar la observación sistemática abierta, una de las técnicas recomendadas por Díaz Nociy Palácios (2009) para estudios de periodismo digital. La principal justificación para el desarrollo de la observación sistemática abierta en la determinación de las categorías de análisis se sostiene en el hecho de que el blog periodístico es un objeto de investigación relativamente nuevo en las investigaciones sobre ciberperiodismo y carece de observación para explorarlo en cuanto a objeto de estudio.

\section{Análisis de contenido Web}

A partir de la observación sistemática abierta, se delimitan las categorías para la realización del análisis de contenido Web de los blogs periodísticos. De acuerdo con la propuesta, este análisis

${ }^{1}$ Las investigaciones periodísticas más recientes suelen aplicar el método incluyendo una observación bien estructurada y automatizada (con el uso de base de datos) o más abierta, con enfoque cualitativo. "Actualmente, en los estudios de comunicación y periodismo, la observación muchas veces se reduce a este tipo especial de método cualitativo, exploratorio (QUANDT, 2008, p.132)”. 
se divide en tres partes, cuyos parámetros para la determinación de las categorías de análisis han sido direccionados de acuerdo con los objetivos específicos de la investigación. Por lo tanto, el análisis de contenido ${ }^{2}$ Web de los j-blogs propuesto en este trabajo se constituye de tres partes, las cuales denominamos: a) análisis de formato; b) análisis de contenido; y c) análisis periodístico.

a) Análisis de formato: se trata de estudiar los elementos que están propiciando la evolución con respecto al diseño y a la estructura de los blogs periodísticos. Además, esta parte del análisis es fundamental para comprender los elementos personalizados por los periodistas-blogueros. Por ello, se divide en cuatro grandes categorías:

- Presentación: 1) Perfil del periodista-bloguero (incluyendo sexo, edad e información sobre la carrera profesional) y 2) Presentación del blog. Ambas subcategorías sirven para averiguar, sobre todo, la experiencia profesional de los periodistas-blogueros y cómo describen la bitácora para la audiencia.

- Contenido: 1) Elementos multimedia; 2) Personalización de contenidos; 3) Uso de imágenes estáticas y dinámicas; 4) Servicios; 5) Documentos archivados y 6) Actualización. Estas categorías contribuyen para verificar los diferentes elementos referentes al contenido, tanto en su almacenamiento y actualización, como en los tipos de contenido que se ofrece en los j-blogs.

- Accesibilidad: 1) Buscador interno/ externo; 2) Normas del blog; 3) Posibilidades de feedback y 4) Hipervínculos. El análisis de las respectivas categorías revelan los elementos de accesibilidad disponibles en cada blog periodístico y si hay un código de conducta para la participación de los usuarios.

\footnotetext{
${ }^{2}$ Algunos investigadores utilizan el análisis de contenido para examinar los blogs como un nuevo género de comunicación (Herring et al., 2004, 2005; Papacharissi, 2004). Otros estudios optan por el análisis de la retórica (MILLER; SHEPHERD, 2004) y la entrevista (NARDI; SCHIANO; GUMBRECHT, 2004) para caracterizar las formas, las funciones y la audiencia de los blogs (HERRING et al., 2006).
} 
- Elementos: 1) Cabecera; 2) Publicidad; 3) Infografía; 4) Integración con redes sociales/ agregadores de noticias; 5) Blogroll y 6) Descripción general del diseño del blog. Esta parte del análisis consiste en hacer una descripción tanto del formato como del diseño de cada j-blog, así como de otros elementos que pueden influir en el contenido informativo, que es la presencia de anuncios publicitarios y la integración a redes sociales.

Al tratarse de elementos que forman parte de aspectos relativos al uso y evolución del formato blog, el análisis de formato revela datos importantes acerca de cada bitácora analizada.

b) Análisis de contenido: corresponde a un análisis cuantitativo que da a conocer algunos datos referentes a los blogs periodísticos, tales como:

- Promedio de entradas por semana.

- Promedio de palabras por post.

- Promedio de palabras por título de cada entrada.

- Promedio de comentarios por entrada.

- Promedio de hiperenlaces por entrada.

- La interpretación de los datos del análisis de contenido Web sirve como complemento por ejemplo para analizar, sobre todo, el estilo de redacción y el nivel de interactividad de los periodistas-blogueros con los usuarios y entre los usuarios.

c) Análisis periodístico: se refiere al análisis de los aspectos propiamente periodísticos, con el objetivo de conocer el estilo de redacción, la narrativa y otros elementos del contenido de los blogs periodísticos. Para ello, se recomienda el análisis de las siguientes categorías:

1. Manual de estilo: para verificar si el periodista-bloguero dispone de un libro de estilo propio, o bien está obligado a seguir el manual de estilo del periódico donde la bitácora se encuentra alojada o no sigue ninguna guía de estilo. En realidad, el objetivo del uso de este material es estandarizar los contenidos, de forma que aparenten cierta homogeneidad en los géneros y en el estilo de redacción 
y edición. En el caso de los blogs periodísticos, la personalización puede conferir al profesional más libertad en cuanto al propio estilo y descartar el uso de un manual.

2. Origen del contenido: a través de esta categoría hemos tratado de averiguar si el contenido del post fue generado por el periodista-bloguero, extraído de otros medios de comunicación o enviado por colaboradores de la bitácora. Así, pues, podemos clasificar la producción en el j-blog según el origen del contenido.

3. Géneros de los posts: el objetivo de analizar los géneros de los contenidos publicados en los blogs periodísticos es identificar los diferentes tipos de géneros: informativos, interpretativos y de opinión. Además, hemos verificado la adaptación y la creación de nuevos géneros ciberperiodísticos ${ }^{3}$. El análisis de los géneros de las entradas se basa en la confección del código por categoría descritos a continuación, que están relacionados con los géneros identificados en las bitácoras analizadas y las referencias bibliográficas consultadas para la elaboración del marco teórico de esta tesis.

\section{Espcificaciones de los géneros}

\section{- Géneros informativos}

El mensaje informativo da a conocer los hechos de la forma más objetiva posible, sin que el periodista exprese ningún juicio. Suele estar relacionado con la noticia. En el caso de los blogs periodísticos, aunque identificamos la presencia del "yo" del profesional, éste no debe emitir opiniones. En caso contrario, el

\footnotetext{
${ }^{3}$ Tras relacionar las referencias bibliográfica sobre los géneros informativos, interpretativos y de opinión, y las que clasifican los diferentes géneros ciberperiodísticos, se ha optado por utilizar el término géneros ciberperiodísticos en el caso de los blogs periodísticos, sobre todo, porque se observa que ya no son los mismos géneros de la prensa escrita. En los j-blogs o en los cibermedios, una noticia puede estructurarse mediante la inserción de recursos hipermedia, lo que no es viable en un periódico. Esa tendencia puede aplicarse en los demás géneros, como la columna de opinión, la crónica, el reportaje etc.
} 
contenido será clasificado como uno de los géneros de opinión. Entre los géneros ciberperiodísticos de información, destacamos los siguientes: noticia, notas informativas, notas cortas tipo móvil y crónica.

Además del encabezado, la noticia suele estructurarse en entradilla o lead - el primer párrafo en el cual se contestan las $5 \mathrm{Ws}$ - y el cuerpo de la noticia. Cuando consta de varios párrafos, es normal que siga un orden decreciente de importancia, más conocido como la técnica de la pirámide invertida.

En cuanto a las notas informativas, son mensajes informativos breves, que actualmente en internet no suelen exceder los 140 caracteres. En el caso de que estas notas informativas cortas sean generadas a partir de un dispositivo móvil, se la denomina notas informativas de tipo móvil. Cuando se publica en la bitácora a través de mensajes cortos enviados desde un teléfono móvil, se trata de una nota informativa de tipo SMS. Si fuera el caso de mensajes enviados al blog vía Twitter, sería otro tipo de mensaje informativo móvil, que se denomina nota informativa corta móvil tipo tweet.

En este caso, la crónica forma parte de los mensajes informativos, sobre todo, porque es un relato elaborado durante un acontecimiento y que cuenta con la presencia física del periodista. Conviene enfatizar que se trata de un relato más objetivo. Los cibermedios también publican la crónica en directo, sea a través de sus Webs o de sus cuentas en Twitter.

\section{- Géneros interpretativos}

La principal característica de los géneros interpretativos es que concede al periodista la oportunidad de interpretar y contextualizar los hechos, excluyendo los juicios de valor. Para esta investigación, hemos tenido en cuenta los siguientes géneros ciberperiodísticos de interpretación:reportaje tradicional, reportaje hipermedia, análisis y entrevista tradicional.

La estructura del reportaje tradicional incluye, además del encabezado, el lead y el cuerpo del reportaje, espacio en el cual se contextualizan e interpretan hechos y datos, que están estructurados en forma de una pirámide invertida (PARRATT, 2003). 
Por ello, es común el uso de infográficos y fotografías para ilustrar un reportaje. Su vinculación temporal con la noticia no es tan cercana como en la crónica. Como una variación del reportaje tradicional, está el reportaje hipermedia, en el que se utilizan recursos multimedia y enlaces para construir la narrativa.

A diferencia del reportaje, el análisis desestructura los hechos y datos con el objetivo de explicar qué ha ocurrido y qué podrá ocurrir, pero sin dejar escapar juicios de valor. En este caso, la noticia pasa a un segundo plano, ya que el análisis asume el papel principal.

Respecto a la entrevista tradicional, se presenta una estructura directa de pregunta-respuesta o indirecta, en forma de texto. Una personalidad suele participar de la entrevista realizada por un periodista, que después hace la transcripción completa y redacta el texto final.

\section{- Géneros de opinión}

Lo que presentan de común los textos de opinión es que trabajan sobre ideas, argumentos y juicios. Entre los diferentes géneros de opinión, incluidos en la presente investigación, están: editorial, artículo firmado, artículo biográfico, columna, comentario y crítica.

El editorial se caracteriza por expresar la línea ideológica del medio de comunicación ante los diferentes temas de actualidad. Corresponde a la voz de la publicación (DÍAZ et al., 1996) y es un texto sin firma.

En el artículo sobresale la firma, que también se suele destacar. Es la firma la que atrae al lector. Se refiere a la colaboración de un experto, periodista o escritor que "colabora con su nombre y su estilo para realzar el prestigio y acentuar la calidad del diario" (GOMIS, 2008, 180). Cada articulista tiene su propio estilo y es libre para analizar y opinar sobre el tema que escribe.

Además del artículo firmado, tenemos el artículo biográfico. Bajo esta categoría se incluyen los textos que analizan o describen la biografía de un personaje público. Hemos preferido no establecer las diferencias entre perfil, semblanza y biografía como sugiere Vilamor (2000, p.405).

A partir del momento en que el artículo aparece como sección y el articulista se convierte en el titular de una rúbrica publicada 
periódicamente, tenemos la columna y el columnista. En cuanto a la estructura, tanto el artículo como la columna dependen del estilo elegido por sus autores.

A pesar de que la mayoría de los investigadores de los géneros periodísticos clasifican el comentario como un gran género de opinión, en esta investigación lo denominamos como un tipo de minicolumna.

La crítica, a su vez, es un texto en el cual se presentan la obra - libro, película, espectáculo etc. - y su autor. Este primer servicio se trata de una información ofrecida al lector, mientras que la descripción de la obra es un segundo servicio (GOMIS, 2008). La crítica periodística es un juicio que interpreta y valora, sin dejar de informar. Una de las variantes, tal y como nos recuerda Santamaría (1997), es la reseña periodística de arte. De carácter informativo, la reseña puede convertirse en una noticia sobre un acontecimiento cultural (concierto, exposición artística, presentación de un libro etc.).

Cuadro 2. Géneros ciberperiodísticos utilizados en el análisis periodístico de losposts de los j-blogs

\begin{tabular}{l|c}
\hline \multicolumn{1}{c}{ Géneros ciberperiodísticos } & Estilo ciberperiodístico \\
\hline Noticia & Información \\
Nota informativa & \\
Nota corta móvil & \\
Crónica & Interpretación \\
\hline Reportaje tradicional & \\
Reportaje hipermedia & \\
Entrevista tradicional & \\
Análisis & \\
\hline Editorial & Opinión \\
Artículo firmado & \\
Artículo biográfico & \\
Columna & \\
Comentario & Dialógico \\
Crítica & Complementario \\
\hline Encuesta digital & \\
\hline Otros contenidos & \\
\hline
\end{tabular}




\section{- Género dialógico}

Los géneros dialógicos posibilitan la conversación y/o la interactividad de los usuarios con los periodistas y otros internautas. La encuesta digital ha sido el único género ciberperiodístico dialógico identificado en los blogs periodísticos analizados. Es un género que se caracteriza por tener una estructura peculiar: una pregunta seguida de dos o más opciones de respuestas, entre las cuales el usuario elige. El resultado se contabiliza automáticamente.

Sin olvidar la importancia de otros géneros periodísticos que no componen la categorización expuesta en esta investigación, como el obituario, el suelto, la reseña, las cartas al director y el encuentro o la entrevista digital, destacamos que estos géneros no han sido identificados en los postspublicados en los blogs periodísticos analizados. A raíz de eso y sin ignorar la importancia que tienen, optamos por no describir dichos géneros periodísticos en la presente tesis doctoral.

\section{- Géneros anexos o complementarios}

El contenido de los posts que no se encuadran en ninguno de los géneros ciberperiodísticos expuestos anteriormente forman parte de los géneros anexos (YANES, 2004) o complementarios (LÓPEZ HIDALGO, 2002). Esta categoría abarca las entradas que han publicado otros tipos de contenido, tales como viñeta, vídeo, música, poesía, discurso oficial etc.

Tras delimitar los criterios correspondientes a los géneros ciberperiodísticos identificados en los diez j-blogs, se ha concretado 16 géneros ciberperiodísticos diferentes encontrados en los 976 postsanalizados, tal y como se presenta en el cuadro 2 . A continuación, se describe las demás categorías del análisis periodístico:

- Estructura del texto: se trata de analizar la estructura del texto de los posts. En conjunción con el promedio de palabras por texto, los datos propiciados por el análisis de esta categoría contribuyen a la identificación de las técnicas utilizadas para elaborar los mensajes informativos e interpretativos. Por ejemplo, se puede confirmar, o no, si los blogs periodísticos redactan noticias utilizando la 
estructura de la pirámide invertida. Además de indicar cómo se estructuran las notas informativas y reportajes. A pesar de contabilizar el número de palabras de los textos de opinión para conocer la extensión que tienen, no nos hemos preocupado de analizar la estructura de estos textos, ya que entendemos que se caracterizan por tener una mayor libertad de estilo.

- Narrativa de los posts: a través de esta categoría hemos identificado los diferentes tipos de narrativas empleadas en las entradas de los blogs periodísticos, que se dividen en: textual, hipertextual, hipermedia y visual. Las entradas que reproducen el estilo de redacción propio de la prensa escrita presentan un lenguaje textual pues ofrecen una lectura lineal. Los posts que incluyen enlaces en el contenido se caracterizan por tener una narrativa hipertextual y proporcionan una lectura multilineal (DÍAZ NOCI, 2001; EDO, 2002; SALAVERRÍA, 2005). Los que además de insertar enlaces también exploran los recursos multimedia, como audio y vídeo, construyen una narrativa hipermedia (SALAVERRÍA, 2005; LARRONDO, 2008). Por último, se encuentran las entradas que publican contenidos visuales, tales como las viñetas.

- Gramática y ortografía: considerando que los blogs, en general, permiten la publicación de cualquier contenido independientemente de la calidad, esta categoría pretende analizar el rigor de los profesionales en aplicar las normas gramaticales a los contenidos publicados. Se trata de verificar, en el texto de los posts, si los periodistas-blogueros han tenido alguna falta ortográfica.

- Número y tipos de fuentes: teniéndose en cuenta que todo relato periodístico debe indicar de forma precisa las fuentes informativas consultadas (MARTÍNEZ ALBERTOS; SANTAMARÍA, 1993) y considerando que las bases de datos y documentos disponibles en internet también sirven de fuentes para periodistas (MENCHER, 2000; MARTÍNEZ-FRESNEDA, 2004), a través de esta cate- 
goría buscamos identificar los tipos de fuentes utilizados por los periodistas-blogueros. En el caso de los j-blogs, sus autores no suelen ser testigos de los hechos que notician. Suelen basarse en informaciones facilitadas por fuentes de segunda mano - cuando un testigo informa sobre lo ocurrido - o de tercera mano, cuando alguien facilita la información a la fuente, y ésta la transmite (MENCHER, 2000). Por ello, nos interesa saber cuáles son las fuentes informativas consultadas por los periodistas-blogueros, que pueden ser la propia observación de un hecho, un testigo, una nota de prensa, el contenido publicado en los cibermedios etc.

- Número, tipo y destino de los enlaces: el hipertexto ofrece al usuario una lectura no lineal, pudiéndose construir diferentes percepciones dependiendo de la trayectoria elegida. Por ello, nos interesa conocer los tipos y los destinos de los enlaces insertados en los postsde las diez bitácoras periodísticas analizadas. Además, nos importa contabilizar el número de enlaces insertados en cada entrada.

- Fomento de la participación: para verificar el nivel de interacción de los periodistas-blogueros con los usuarios en el espacio de comentarios del blog. Este apartado fundamenta su importancia en la identificación del dialogo entre el profesional y la audiencia, comentando en este espacio y fomentando la participación ciudadana en la bitácora.

- Capacidad de producción: conocer cuántos periodistas trabajan en la generación de contenidos del blog es el objetivo de esta categoría. Al diseñar la base de datos para la realización del análisis de contenido Web de los j-blogs, se recomienda destinar un espacio para anotar observaciones acerca del autor de cada post. Así, pues, se conoce el número de periodistas que desarrollan contenidos en estas bitácoras, ya que hay blogs donde más de un periodista actualiza el contenido. 


\section{Entrevista con los periodistas-blogueros}

Con el objetivo de complementar y contrastar las informaciones y datos obtenidos durante las tres etapas del análisis de contenido Web de los blogs periodísticos, se sugiere la realización de entrevistas en profundidad semiabierta con los periodistas-blogueros. Se considera que recurrir a esta técnica es fundamental para este tipo de investigación, ya que se puede conseguir informaciones directamente con los periodistas-blogueros y compararlas con los resultados del análisis. Además, las entrevistas pueden aportar información no aclarada en los análisis.

Esta comparación se convierte en una etapa importante para la comprensión del universo del contenido de los blogs periodísticos y del contexto donde están insertados. Según Duarte, la "entrevista en profundidad es un recurso metodológico que busca, con base en teorías y presupuestos definidos por el investigador, recoger respuestas a partir de la experiencia subjetiva de una fuente" (2005, p.62).

A través de la entrevista en profundidad se puede comprender varios aspectos: cómo los periodistas perciben un producto informativo; explicar la producción de noticias en un medio de comunicación; identificar las principales fuentes de información de periodistas de una sección etc. El guión de las entrevistas realizadas con los periodistas-blogueros se debe basar en los resultados del análisis obtenido en cada caso específico.

\section{Recogida de datos}

Para sistematizar el análisis, se propone la construcción de una base de datos para recoger los datos y, a continuación, interpretarlos. La base de datos puede seguir una estructura similar a la que aparece en la figura 1. En este caso, se ha creado tres apartados diferentes, siguiendo las categorías propuestas en los tres análisis que componen el análisis de contenido Web de los blogs periodísticos. El apartado "Posts" corresponde al espacio para registrar los datos referentes a cada entrada, que forma parte del análisis 
periodístico. En "Datos del blog”, se registra la información general relativa al periodista-bloguero y al blog. Por último, en "análisis formato", se anota las especificidades de cada $j$-blog en cuanto a los elementos de formato.

Figura 1. Base de datos para recogida de información de cada blog/post

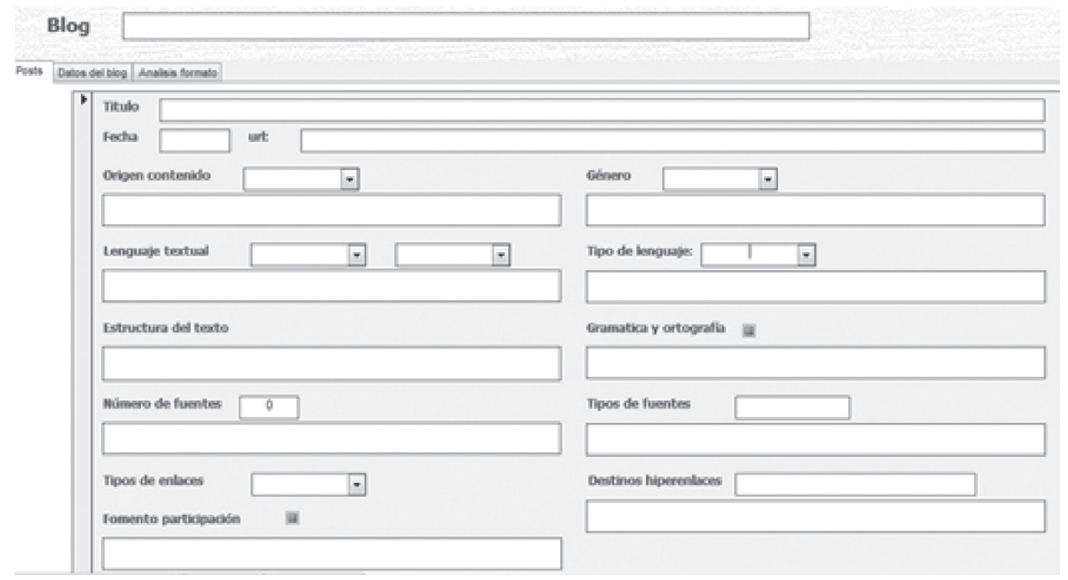

El uso de una base de datos creada específicamente para una investigación facilita la organización del trabajo, además de sistematizar la recogida y el tratamiento de los datos. Sin el uso de esta herramienta en un estudio sobre blogs periodísticos en que se analizan los posts, el tratamiento de los datos no ocurriría con la misma eficacia.

\section{Consideraciones finales}

Esta propuesta metodológica reúne diferentes técnicas de investigación para el análisis de blogs periodísticos, que se divide en tres diferentes tipos de análisis (formato, contenido y periodístico), dentro de lo que Herring (2010) denomina análisis de contenido Web. Además, a través de la definición de las variables de análisis, se consigue definir categorías de acuerdo con los objetivos específicos de cada investigación. 
De acuerdo con la investigación empírica a la cual se ha puesto a prueba la presente propuesta metodológica, conviene destacar que no siempre el análisis de contenido Web es suficiente para responder a todas las preguntas de la investigación. Por ello, se considera que la entrevista en profundidad con los autores de las bitácoras aclara aspectos relevantes de los resultados del análisis. La entrevista con el periodista-bloguero también apunta datos que el análisis puede no hacer referencia, tales como el motivo por el cual prefiere mantener determinados elementos en su bitácora o qué criterios utiliza para contestar el comentario escrito por un usuario sobre un artículo publicado en el blog. Las entrevistas complementan el análisis de contenido Web, de carácter mayoritariamente cuantitativo, dando a la investigación un carácter más cualitativo.

Cabe subrayar que el diseño de una propuesta metodológica específica para la realización de un estudio exploratorio del blog periodístico es determinante para conocer los elementos del objeto de investigación en profundidad. No es lo mismo adaptar metodologías desarrolladas para el análisis de cibermedios, ya que los blogs tienen sus peculiaridades en cuanto objeto de estudio que necesitan ser investigadas.

Por último, se destaca que la construcción de una base de datos específica para el estudio de los blogs periodísticos se convierte en una herramienta fundamental para realizar el tratamiento de los datos con eficacia. Con los datos insertados, los gráficos y tablas se generan de forma automatizada, lo que evita errores de contabilidad. No menos importante es la interpretación que el investigador hará de esos datos.

\section{Referencias}

ARAÚJO, Artur. Weblog e jornalismo: os casos de No Mínimo Weblog e Observatório da Imprensa (Bloi), 2006. 582 f. Dissertação (Mestrado em Ciências da Comunicação - Jornalismo). Escola de Comunicação e Artes, Universidade de São Paulo. 
COLUSSI RIBEIRO, Juliana. El blog periodístico como mini diario digital. 2013. 494 f. Tesis (Doctorado en Periodismo). Facultad de Ciencias de la Información, Universidad Complutense de Madrid.

DÍAZ NOCI, Javieret al. El periodismo electrónico. Información y servicios multimedia en la era del ciberespacio. Barcelona: Ariel, 1996

. La escritura digital: hipertexto y construcción del discurso informativo en el periodismo electrónico. Bilbao: Servicio Editorial de la Universidad del País Vasco, 2001.

; PALÁCIOS, Marcos (eds.).Ciberperiodismo: métodos de investigación. Una aproximación multidisciplinar en perspectiva comparada. Bilbao: Servicio Editorial de la Universidad del País Vasco, 2009.

DOMINGO, David, HEINONEN, Ari. Weblogs and journalism.A typology to explore the blurring boundaries. Nordicom Review, v. 29, p.3-15, 2008.

DUARTE, Jorge. Entrevista em profundidade. In: DUARTE, Jorge. (org.).Métodos e Técnicas de Pesquisa em Comunicação.São Paulo:Atlas, v. 1, 1ํe ed., p. 62-83, 2005.

EDO, Concha. Del papel a la pantalla: la prensa en Internet. Sevilla: Comunicación social, 2002.

FOLETTO, Leonardo F.O blog jornalístico: definição e características na blogosfera brasileira.2009. 161 f. Dissertação (Mestrado em Jornalismo). Centro de Comunicação e Expressão,Universidade Federal de Santa Catarina.

FRAGOSO, Suely. et al.Métodos de pesquisa para Internet. Porto Alegre: Sulina, 2011.

GOMIS, Lorenzo.Teoría de los géneros periodísticos.Barcelona: UOC, 2008.

HERRING, Susan. Web contentanalysis: Expanding the paradigma. In: HUNSINGER, Jeremy. et al. (eds.): International Handbookof Internet Research. London: Springer Verlag, 2010. p.233-249.

LARRONDO URETA, Ainara.Los géneros en la redacción ciberperiodística. Contexto, teoría y práctica actual.Bilbao: Servicio Editorial de la Universidad del País Vasco, 2008.

LÖFFELHOLZ, Martín; WEAVER, David.Questioning national, cultural and disciplinary boundaries: A call for global journalism research.In: LÖFFE- 
LHOLZ, M.; D. WEAVER (eds.) Global journalism research. Theories, Methods, findings, Future. Cambridge, Blackwell Publishing, 2008. p.233-249.

LÓPEZ HIDALGO, Antonio. Géneros periodísticos complementarios. Sevilla: Comunicación Social, 2002.

MARCONI, Marina de A., LAKATOS, Eva M. Fundamentos de metodologia científica. São Paulo: Atlas, 2004.

MARTÍNEZ ALBERTOS, José Luís; SANTAMARÍA, Luisa. Manual de estilo. Indianápolis:Inter American PressBooks, 1993.

MARTÍNEZ-FRESNEDA, Humberto.Las fuentes en el periodismo informativo. In: CANTAVELLA, Juan, SERRANO; José F.Redacción para periodistas: informar e interpretar. Madrid, Ariel, 2004. p.233-249.

MENCHER, Melvin.News Reporting and Writing.Boston: McGraw-Hill, 8a ed., 2000.

MESO, Koldo et al.Ferramenta para análise de blogs em cibermeios. In: PALÁCIOS, Marcos (Org.) Ferramentas para análise de qualidade no ciberjornalismo.Covilhã:Labcom, 2011. p. 51-80.

NOGUERA VIVO, José Manuel. La bitácora como producto ciberperiodístico y corporativo de los medios de comunicación: aproximación a las relaciones de los cibermedios con la comunidad bloguer. 2006. 454 f. Tesis (Doctorado). Departamento de Ciencias Sociales, Jurídicas y de la Empresa, Universidad Católica San Antonio.

PARRAT, Sonia. Introducción al reportaje: antecedentes, actualidad y perspectivas.Santiago de Compostela: Servicio de Publicacións da USC, 2003.

QUANDT, Thorsten. Methods of journalism research - Observation. In: LÖFFELHOLZ, Martín, WEAVER, David (eds.).Global journalism research. Theories, Methods, findings, Future. Cambridge: Blackwell Publishing, 2008. p.131-141.

RECUERO, Raquel.Warblogs: Os Blogs, a Guerra no Iraque e o Jornalismo Online. Disponível em: http://pontomidia.com.br/raquel/warblogs.pdf. Acesso em: 5 out. 2010 .

ROBINSON, Susan. The mission of the j-blog.Recapturing journalistic authority online. Journalism, v. 7, n.1. p.65-83, 2006. 
SALAVERRÍA, Ramón. Redacción periodística en Internet. Navarra: Eunsa, 2005.

SANTAMARÍA, Luisa.Géneros para la persuasión en periodismo. Madrid: Fragua, 1997.

SINGER, Jane.The political j-blogger.'Normalizing' a new media form to fit old norms and practices. Journalism, v. 6, n. 2, p. 173-198, 2005.

VILAMOR, José R. Redacción periodística para la generación digital. Madrid: Editorial Universitas, 2000.

YANES MESA, Rafael. Géneros periodísticos y géneros anexos. Madrid, Fragua, 2004.

\section{Juliana Colussi Ribeiro}

Es doctora y máster en Periodismo por la Universidad Complutense de Madrid (Espanha). Máster en Comunicação Midiática y Periodista por la Universidade Estadual Paulista (Brasil). Tiene experiencia en prensa escrita y gabinetes de prensa tanto en Brasil como en España. Fue profesora en diferentes universidades privadas de Brasilia.

Recebido em: 13.08.2013

Aceito em: 09.11.2013 